\title{
Luminescence decay in highly excited GaN grown by hydride vapor-phase epitaxy
}

S. Juršènas, S. Miasojedovas, G. Kurilčik, and A. ŽukauskasP. R. Hageman

Citation: Appl. Phys. Lett. 83, 66 (2003); doi: 10.1063/1.1590736

View online: http://dx.doi.org/10.1063/1.1590736

View Table of Contents: http://aip.scitation.org/toc/apl/83/1

Published by the American Institute of Physics

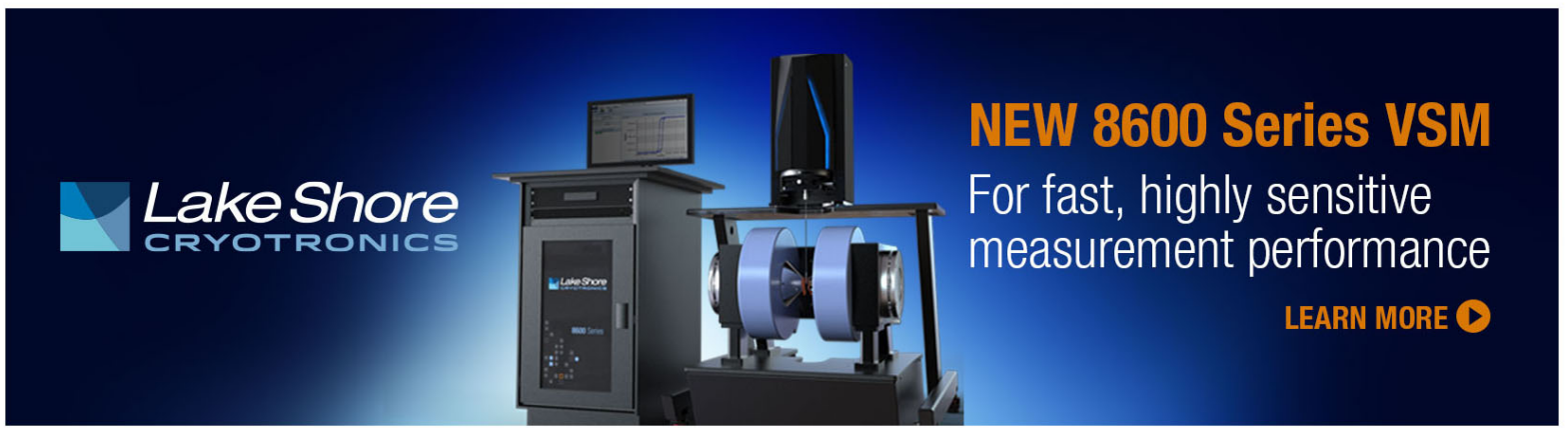




\title{
Luminescence decay in highly excited GaN grown by hydride vapor-phase epitaxy
}

\author{
S. Juršènas, ${ }^{\text {a) }}$ S. Miasojedovas, G. Kurilčik, and A. Žukauskas \\ Institute of Materials Science and Applied Research, Vilnius University, Sauletekio 9-III, LT-2040 Vilnius, \\ Lithuania \\ P. R. Hageman \\ University of Nijmegen, Faculty of Science, Department of Exp. Solid State Physics III, Toernooiveld 1, \\ 6525 ED Nijmegen, The Netherlands
}

(Received 15 April 2003; accepted 9 May 2003)

\begin{abstract}
Carrier recombination dynamics in GaN grown by hydride vapor-phase epitaxy has been studied by means of transient photoluminescence under high photoexcitation conditions that are close to stimulated emission regime. The luminescence transient featured an exponential decay with the time constant of $205 \mathrm{ps}$ at room temperature. The transient was shown to be in good agreement with a model of saturated centers of nonradiative recombination with the trap density of $\sim 10^{17} \mathrm{~cm}^{-3}$ and carrier recombination coefficients of $\sim 10^{-8} \mathrm{~cm}^{3} / \mathrm{s}$. In such a regime, the lifetimes of electrons and holes have a common value of 410 ps. (C) 2003 American Institute of Physics.
\end{abstract}

[DOI: $10.1063 / 1.1590736$ ]

Formation of high-quality bulk-like GaN layers is of great importance since they are used as substrates for further epitaxy of light emitting structures and can facilitate efficient light extraction from light emitting diodes. ${ }^{1,2}$ Conventional heteroepitaxial growth of $\mathrm{GaN}$ on low temperature $\mathrm{GaN}$ or AlN buffer layers deposited on $\mathrm{Al}_{2} \mathrm{O}_{3}$ and $\mathrm{SiC}$ substrates results in films containing high dislocation densities $\left(10^{8}-10^{10} \mathrm{~cm}^{-2}\right)$ due to lattice mismatch between the film and the substrate. These threading dislocations affect both optical and electrical properties and are believed to hinder the improving of device performance. The best quality GaN epilayers are obtained by using homoepitaxial growth over bulk GaN crystals. ${ }^{3,4}$ However, at present, the size and growth rate of the high-pressure crystals are limited. Hydride vapor phase epitaxy (HVPE) offers large growth rates with relatively good quality of crystals. ${ }^{5,6}$ However, optical properties under high excitation conditions, which are important for establishment of laser and high-power light emitting diode operation regime, are not well investigated in HVPE grown GaN. Recently, we have shown that in highly excited $\mathrm{GaN}$, deep-trap saturation can occur and luminescence transient can provide a common electron and hole lifetime, which is one of the crucial indicators of the materials quality. ${ }^{7}$ Here we present estimations of carrier lifetime in HVPE grown $\mathrm{GaN}$ under high excitation.

The nominally undoped $\mathrm{GaN}$ film was deposited in a home built, horizontal HVPE reactor using a metalorganic chemical vapor deposition (MOCVD)-grown GaN layer $(\sim 1.9 \mu \mathrm{m})$ on a sapphire substrate as a template. ${ }^{8}$ The HVPE reactor was equipped with a two-zone furnace and operated at atmospheric pressure. Nitrogen was used as a carrier gas (1 slm) and $\mathrm{NH}_{3}$ as a nitrogen precursor $(400 \mathrm{sccm})$. The reactor was designed to obtain laminar flow and allows mixing of the $\mathrm{NH}_{3}$ and the $\mathrm{GaCl}$ just above the susceptor. The $\mathrm{GaCl}$ growth species were in situ synthesized by passing

${ }^{a)}$ Electronic mail: saulius.jursenas@ff.vu.lt pure $\mathrm{HCl}$ over liquid gallium $(7 \mathrm{~N})$ at $800^{\circ} \mathrm{C}$. At a growth temperature of $955^{\circ} \mathrm{C}$, we obtained a layer thickness of 105 $\mu \mathrm{m}$ for a growth time of $1 \mathrm{~h}$ using $40 \mathrm{sccm} \mathrm{HCl}$. The equilibrium electron density was estimated to be $n_{0}=5$ $\times 10^{16} \mathrm{~cm}^{-3}$.

The sample was excited by the fourth harmonic (photon energy $h \nu_{g}=4.66 \mathrm{eV}$ ) of the actively-passively mode-locked yttrium-aluminum-garnet $\mathrm{Nd}^{3+}$ laser (pulse duration of $\tau_{g}=20 \mathrm{ps}$, repetition rate $2.7 \mathrm{~Hz}$, maximum pump energy 20 $\mu \mathrm{J})$. The size of the excitation spot was approximately $1 \mathrm{~mm}$. Luminescence was collected in backward geometry and dispersed by a $0.4 \mathrm{~m}$ grating monochromator. Toluene optical Kerr shutter was used for temporal resolution $(20 \mathrm{ps})$ of the luminescence. The experiments were carried out at room temperature.

Figure 1 displays typical time-resolved luminescence spectra of the HVPE grown GaN for an excitation energy density of $I_{g}=1.2 \mathrm{~mJ} / \mathrm{cm}^{2}$. The spectra are seen to contain one broad emission band peaked in the vicinity of the bandgap energy $(\sim 3.4 \mathrm{eV})$. The band becomes narrower and redshifts with time. Figure 2 displays the time-integrated luminescence spectrum and the spectral dependence of the instantaneous decay time. The luminescence decay time is seen to be strongly dependent on the photon energy with a highest value at the peak of the luminescence spectrum and a decrease at both the high- and low-energy wings of the band. The observed luminescence dynamics is typical of the radiative recombination of high-density electron-hole plasma $(\mathrm{EHP})^{7,9}$ that occurs under intense photoexcitation with the excitonic states screened by the carrier system (for a thickness of the excited region of $d_{g} \approx 0.1 \mu \mathrm{m},{ }^{9}$ the estimated initial carrier density is $\sim 10^{19} \mathrm{~cm}^{-3}$, i.e., essentially higher than the Mott density). ${ }^{9,10}$ Narrowing of the EHP band with time is due to decrease in carrier density and, predominantly, due to decrease in temperature of plasma and longwavelength optical phonons. ${ }^{711-13}$ Analysis of the highenergy wing of the recorded EHP band within a simple one- 


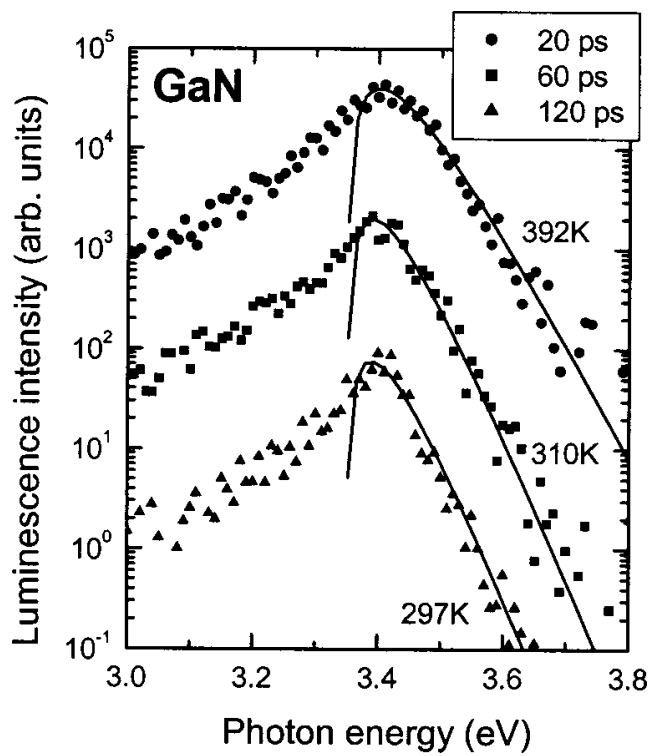

FIG. 1. Luminescence spectra of a HVPE grown GaN film for the excitation density $I_{g}=1.2 \mathrm{~mJ} / \mathrm{cm}^{2}$ recorded for backward geometry at different delay time. The spectra are arbitrarily shifted along the vertical axis. Points, experiment; solid lines, calculation. The deduced carrier temperature is indicated at each spectrum.

particle approach ${ }^{14}$ (simulated spectra are shown by lines in Fig. 1) indicates that the carrier temperature reaches a value of $392 \mathrm{~K}$ at zero delay and relaxes exactly to the equilibrium value in the first $100 \mathrm{ps}$. It should be noted that owing to a small thickness of the excited region, the radiation that escapes perpendicularly to the surface is mainly due to spontaneous emission of EHP. However, laterally stimulated emission may also occur resulting in reduced carrier lifetime on the initial decay stage. ${ }^{7}$

Open points in Fig. 3 depict variation of the luminescence intensity at the peak position of the emission band (3.4 $\mathrm{eV}$ ) for $I_{g}=1.2 \mathrm{~mJ} / \mathrm{cm}^{2}$. The luminescence decay is seen to be almost exponential with a time constant of $\tau_{\mathrm{LUM}}=205 \mathrm{ps}$. Increased excitation intensity $\left(I_{g}=3 \mathrm{~mJ} / \mathrm{cm}^{2}\right.$; solid points in Fig. 3) results in an occurrence of nonexponential decay on the initial stage of relaxation $(t<100 \mathrm{ps})$. However, in the later stage of the relaxation, the exponential decay yields the same value of the time constant.

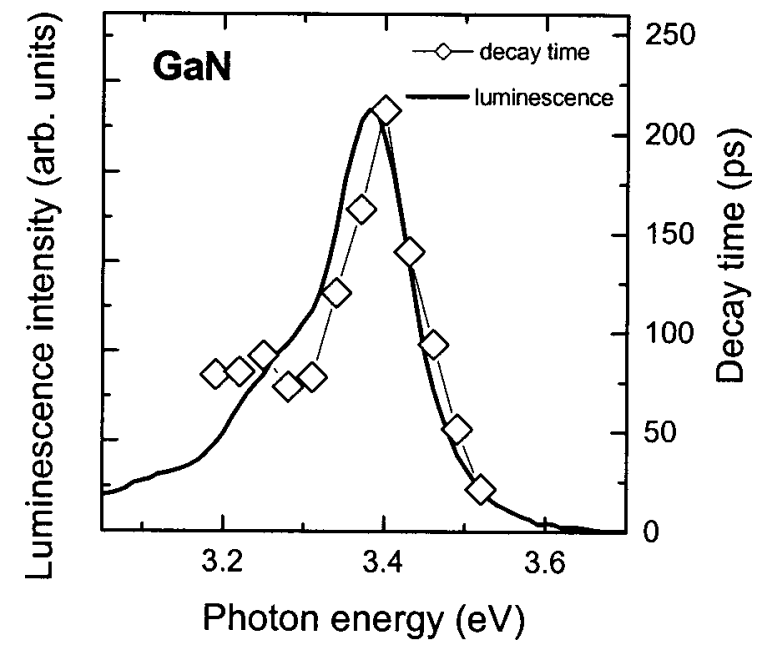

FIG. 2. Time-integrated luminescence spectrum of HVPE grown GaN film (line) and spectral dependence of the instantaneous decay time (points).

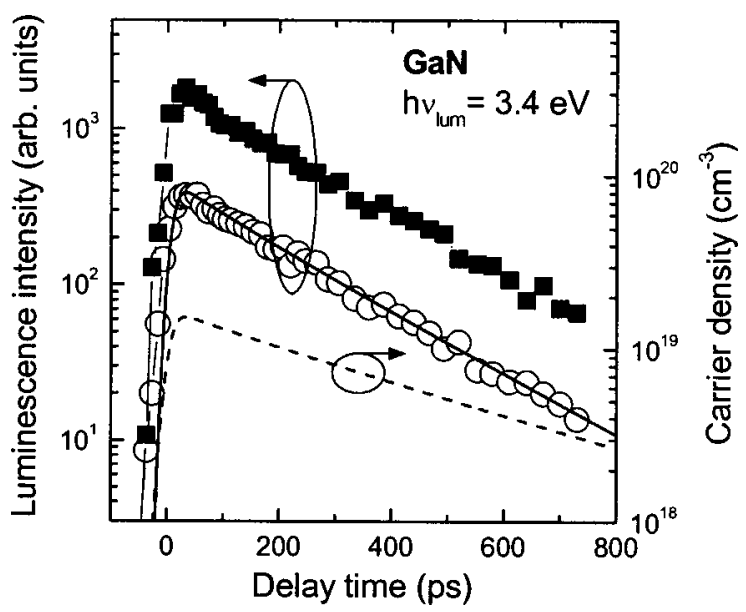

FIG. 3. Transient behavior of the luminescence intensity at $3.4 \mathrm{eV}$ photon energy for two excitation densities $I_{g}=1.2 \mathrm{~mJ} / \mathrm{cm}^{2}$ and $I_{g}=3 \mathrm{~mJ} / \mathrm{cm}^{2}$ (open and solid points, respectively). Solid line, calculated luminescence intensity; dashed line, calculated carrier density for $I_{g}=1.2 \mathrm{~mJ} / \mathrm{cm}^{2}$.

The decay rate of the band-to-band luminescence in a semiconductor is related to the carrier lifetime, however, it depends on the photoexcitation intensity in a complex manner, and rely on several factors such as population of the capture traps by electrons and holes, plasma heating and degeneration, and various multiparticle interactions. ${ }^{15}$

To account for the observed luminescence transients, consider a typical model for spontaneous carrier recombination ${ }^{16}$ for an $n$-type crystal $\left(n_{0} \gg p_{0}\right)$. For simplicity, we assume one dominant type of capture centers with the density $N_{t}$ and recombination coefficients $b_{e}$ and $b_{h}$ for electrons and holes, respectively. Neglecting excitonic effects, carrier diffusion and photon recycling, the temporal evolution of the concentration of electrons, holes, and recombination centers is governed by the following set of differential equations:

$$
\begin{aligned}
& d n / d t=G(t)-b_{r}\left(\Delta n+n_{0}\right) \Delta p-b_{e} n_{t}^{+}\left(\Delta n+n_{0}\right), \\
& d p / d t=G(t)-b_{r}\left(\Delta n+n_{0}\right) \Delta p-b_{h} n_{t}^{0} \Delta p, \\
& d n_{t}^{+} / d t=-b_{e} n_{t}^{+}\left(\Delta n+n_{0}\right)+b_{h} n_{t}^{0} \Delta p,
\end{aligned}
$$

where $G(t)$ is the generation rate, $n, p, \Delta n$, and $\Delta p$, are the total concentrations and the excess densities of electrons and holes, respectively, with $n=n_{0}+\Delta n$ and $p \approx \Delta p ; n_{t}^{+}$and $n_{t}^{0}$ are the concentrations of the filled and empty recombination centers, respectively, with $n_{t}^{+}+n_{t}^{0}=N_{t}$; and $b_{r}$ is the coefficient of bimolecular recombination. The luminescence yield due to band-to-band recombination is

$$
I_{\mathrm{LUM}}(t)=b_{r} \Delta p(t)\left[\Delta n(t)+n_{0}\right] .
$$

In such an approach, depending on the excitation density, the luminescence decay transient can be described under one of several limiting conditions. For typical asymmetry of the capture cross sections (say, $b_{h}>b_{e}$ ) and a low excitation intensity $\left(\Delta p \ll N_{t}\right)$ the traps are almost empty $\left(n_{t}^{+} \ll N_{t}\right)$, the hole density decays with a time constant $\tau_{h}=\left(b_{h} N_{t}\right)^{-1}$, and the electron density varies much slower. This results in an exponential luminescence decay with a time constant of $\tau_{\text {LUM }}=\tau_{h}$. At higher excitation intensities $\left(\Delta p \gg N_{t}, \Delta n\right.$ $\left.\gg n_{0}\right)$, the traps are saturated by holes $\left(n_{t}^{0} \ll N_{t}\right)$ and the car- 
rier recombination is controlled by the electron capture time $\tau_{e}=\left(b_{e} N_{t}\right)^{-1}$ provided that the bimolecular recombination rate is negligible $\left(b_{r} n \ll b_{e} N_{t}\right)$. Since holes are captured only by empty traps, the hole lifetime is increased up to the electron lifetime. This means that EHP can be characterized by a common carrier density $n \approx p$ and a common lifetime $\tau_{e}$ $\approx \tau_{h}$. Consequently, Eq. (4) yields an exponential decay with a time constant of $\tau_{\mathrm{LUM}} \cong \tau_{e} / 2$. With further increase of the plasma density when $b_{r} n_{e} \gg b_{e} N_{t}$, the luminescence decay becomes nonexponential and plasma-density dependent.

Since the observed exponential luminescence decay rate is almost independent of the excitation density, to account for the experimental results one should chose between two highexcitation limiting regimes of either unsaturated or saturated traps. ${ }^{16}$ In the unsaturated-trap regime, the concentration of the traps should be in the range of $10^{20} \mathrm{~cm}^{-3}$ to preserve the condition $\Delta p \ll N_{t}$. Consequently, the hole recombination coefficient should take a value below $1 \times 10^{-10} \mathrm{~cm}^{-3}$. For the room-temperature thermal velocity of holes in $\mathrm{GaN}$ $\left(\approx 10^{7} \mathrm{~cm} / \mathrm{s}\right)$, this yields a hole capture cross section below $10^{-18} \mathrm{~cm}^{-3}$. For electrons, the capture cross section should be even smaller $\left(\sim 10^{-19} \mathrm{~cm}^{-3}\right)$ because of the condition $b_{e}<b_{h}$ and a higher thermal velocity. These cross sections are of 3-4 orders smaller than typical cross sections of point defects $\left(\approx 10^{-15} \mathrm{~cm}^{2}\right)$ and are difficult to explain even by high barriers caused by configuration and repulsive Coulomb potential, since, typically, tunneling through the barriers allow no decrease of the capture cross sections by more than two orders.

Much more realistic picture appears under assumption of the saturated-trap regime. Now, to account for the exponential decay on the very late stage of the observed relaxation when the carrier density is around $10^{18} \mathrm{~cm}^{-3}$, the trap density should not exceed $\sim 10^{17} \mathrm{~cm}^{-3}$. This yields an electron recombination coefficient of at least $1 \times 10^{-8} \mathrm{~cm}^{-3}$ and higher values for the hole recombination coefficient. For the room-temperature thermal velocities, these values are in good agreement with the capture cross sections of the order $10^{-15} \mathrm{~cm}^{2}$ and higher (higher values of the capture cross sections are plausible in $\mathrm{GaN}$ because of the presence of extended defects such as threading dislocations). Line in Fig. 3 depicts the results for the luminescence transient simulation with $b_{n}=1 \times 10^{-8} \mathrm{~cm}^{3} / \mathrm{s}$ and $b_{p}=1 \times 10^{-7} \mathrm{~cm}^{3} / \mathrm{s}$. A value of $3 \times 10^{-11} \mathrm{~cm}^{3} / \mathrm{s}$ was used for the bimolecular recombination coefficient (an average of the data reviewed in Ref. 17). To fit the exponential decay with the experimental points, the density of traps was set to a value of $N_{t}=2.1$ $\times 10^{17} \mathrm{~cm}^{-3}$. Dashed line in Fig. 3 shows the calculated temporal dependence of the electron density that proves the high-excitation condition $\left(\Delta n \gg N_{t}, n_{0}\right)$ to be safely satisfied.

In conclusion, temporal evolution of dense EHP recombination was studied in a HVPE grown GaN epilayer under high-excitation conditions close to the carrier degeneration threshold $\left(n \cong 10^{19} \mathrm{~cm}^{-3}\right)$. The luminescence decay transient was shown to be in good agreement with a model of saturated centers of nonradiative recombination with the trap density of $\sim 10^{17} \mathrm{~cm}^{-3}$ and carrier recombination coefficients of $\sim 10^{-8} \mathrm{~cm}^{3} / \mathrm{s}$. In such a regime, the luminescence decay time of 205 ps yields a common lifetime of 410 ps for both electrons and holes. This value is smaller than in homoepitaxialy grown samples, but it is considerably higher than in high quality MOCVD grown epilayers grown on sapphire. ${ }^{7}$ This suggests high potential of HVPE grown GaN for short-wavelength optoelectronics applications.

The research at Vilnius University was partially supported by the Lithuanian State Science and Education Foundation and European Commission supported SELITEC center Contract No. G5MA-CT-2002-04047. A.Ž. acknowledges the Lithuanian Ministry of Education and Science for his Fellowship.

${ }^{1}$ S. Nakamura and G. Fasol, The Blue Laser Diode: GaN Based Light Emitters and Lasers (Springer, Berlin, 1997).

${ }^{2}$ A. Zukauskas, M. S. Shur, and R. Gaska, Introduction to Solid-State Lighting (Wiley, New York, 2002).

${ }^{3}$ C. Kirchner, V. Schwegler, F. Eberhard, M. Kamp, K. J. Ebeling, K. Kornitzer, T. Ebner, K. Thonke, R. Sauer, P. Prystawko, M. Leszczynski, I. Grzegory, and S. Porowski, Appl. Phys. Lett. 75, 1098 (1999).

${ }^{4}$ M. Leszczynski, B. Beaumont, E. Frayssinet, W. Knap, P. Prystawko, T. Suski, I. Grzegory, and S. Porowski, Appl. Phys. Lett. 75, 1276 (1999).

${ }^{5}$ G. E. Bunea, W. D. Herzog, M. S. Ünlü, B. B. Goldberg, and R. J. Molnar, Appl. Phys. Lett. 75, 838 (1999).

${ }^{6}$ P. W. Yu, C. S. Park, and S. T. Kim, J. Appl. Phys. 89, 1692 (2001).

${ }^{7}$ S. Juršenas, N. Kurilčik, G. Kurilčik, A. Žukauskas, P. Prystawko, M. Leszczynski, T. Suski, P. Perlin, I. Grzegory, and S. Porowski, Appl. Phys. Lett. 78, 3776 (2001).

${ }^{8}$ V. Kirilyuk, P. R. Hageman, P. C. M. Christianen, P. K. Larsen, and M. Zielinski, Appl. Phys. Lett. 79, 4109 (2001).

${ }^{9}$ F. Binet, J. Y. Duboz, J. Off, and F. Scholz, Phys. Rev. B 60, 4715 (1999).

${ }^{10}$ S. Hess, R. A. Taylor, J. F. Ryan, B. Beaumont, and P. Gibart, Appl. Phys. Lett. 73, 199 (1998).

${ }^{11}$ J. F. Müller and H. Haug, J. Lumin. 37, 97 (1987).

${ }^{12}$ E. A. Meneses, N. Jannuzzi, J. G. P. Ramos, R. Luzzi, and R. C. C. Leite, Phys. Rev. B 11, 2213 (1975).

${ }^{13}$ S. Juršenas, G. Kurilčik, and A. Žukauskas, Phys. Rev. B 58, 12937 (1998).

${ }^{14}$ G. Lasher and F. Stern, Phys. Rev. 133, 553 (1964).

${ }^{15}$ O. Brandt, J. Ringling, K. H. Ploog, H.-J. Wünsche, and F. Henneberger, Phys. Rev. B 58, R15977 (1998).

${ }^{16}$ O. Brandt, H. Yang, and K. H. Ploog, Phys. Rev. B 54, R5215 (1996).

${ }^{17}$ A. Dmitriev and A. Oruzheinikov, J. Appl. Phys. 86, 3241 (1999). 